\title{
SMART oder MOTTO? Von der Hemmung in die Handlungsfähigkeit durch passende Zielformulierung
}

\author{
Christina Mühlberger • Angela Büche $\cdot$ Eva Jonas
}

Online publiziert: 4. Mai 2018

(C) Der/die Autor(en) 2018

Zusammenfassung Ziele werden in Beratungen oft so konkret wie möglich auf Verhaltensebene formuliert. Ist eine Person jedoch unsicher und besorgt, wenn sie über ihr Ziel spricht, kann davon ausgegangen werden, dass die Identifikation mit dem Ziel zu gering ist, als dass es handlungswirksam werden könnte. Eine Ursache können Konflikte zwischen bewussten und unbewussten Bedürfnissen sein. Die vom Zürcher Ressourcen Modell (ZRM) entwickelten MOTTO-Ziele bringen Bewusstes mit Unbewusstem in Einklang. Sie stellen Zielidentifikation her und können so die Zielverfolgung unterstützen. Identifiziert sich eine Person bereits mit ihrem Ziel, kann sofort mit der konkreten Planung begonnen werden.

Schlüsselwörter Zielformulierung · MOTTO-Ziele · Annäherungsmotivation

\section{SMART or MOTTO? Suitable goal setting to get from avoidance to the ability to act}

\begin{abstract}
In consultancies, goals are often set very concrete on a behavioral level which can increase one's probability of success to attain the goal. However, if people are anxious and worried when they talk about their goals, there seems to be too little identification with the goals and attainment is impossible. The reason is often a conflict between conscious and unconscious needs. The Zürcher Ressourcen
\end{abstract}

\footnotetext{
Mag. Dr. C. Mühlberger $(\bowtie) \cdot$ Prof. Dr. E. Jonas

Fachbereich Psychologie, Universität Salzburg, Hellbrunnerstraße 34, 5020 Salzburg, Österreich

E-Mail: christina.muehlberger@sbg.ac.at

Prof. Dr. E. Jonas

E-Mail: eva.jonas@sbg.ac.at
}

\section{A. Büche, MSc.}

Maierwiesweg 20, 5023 Salzburg, Österreich

E-Mail: angela.bueche@stimmig-leben.com 
Modell (ZRM) has developed MOTTO-goals which are defined on an attitudinal level and bring into accordance consciousness and unconsciousness. They build identification with goals and thus, support goal pursuit. If people already identify with their goals, they can immediately start with concrete planning.

Keywords Goal setting $\cdot$ MOTTO-goals $\cdot$ Approach motivation

Fallbeispiel Es ist Neujahr, und Raketen fiegen in den Himmel. Die 32-jährige Erika ist verheiratet, hat zwei Kinder und arbeitet seit mehr als 5 Jahren in einem Versicherungsunternehmen als Kundenberaterin. Heute nimmt sie sich fest vor, ihre Chefin im kommenden Jahr endlich um eine Beförderung zu bitten. Sie möchte im Gespräch selbstbewusst auftreten und ihre Argumente für einen beruflichen Aufstieg selbstsicher präsentieren. Ein halbes Jahr vergeht, und Erika verschiebt ihren Vorsatz auf den späten Sommer. Doch ehe sie sich versieht, ist das Jahr vorbei, und das Gespräch wird erneut zu ihrem Neujahrsvorsatz.

Wer kennt dies nicht? Die Pläne sind vorhanden, wir sind überaus motiviert, diese umzusetzen, aber am Ende schaffen wir es doch nicht. Laut einer Umfrage der University of Scranton aus dem Jahre 2014 brach ein Viertel der Befragten bereits nach zwei Wochen ihre Neujahrsvorsätze. Sechs Monate später hielt sich nur noch weniger als die Hälfte an ihre Vorsätze. Woran kann es liegen, dass Menschen so häufig daran scheitern, ihre Ziele zu verfolgen? Die psychologische Forschung sagt, dass es zum einen eine große Rolle spielt, wie wir Ziele formulieren, und zum anderen wichtig ist, dass die Zielformulierung zur Persönlichkeit passt.

\section{Zielformulierung}

Ziele können auf unterschiedlichen Ebenen formuliert werden (Storch 2008) - auf der Ergebnis-, Verhaltens- und Haltungsebene. Auf der Ergebnisebene beschreibt die Person, welches Ziel sie erreichen möchte. Erika, beispielsweise, möchte im Gespräch mit ihrer Chefin eine Beförderung erreichen. Auf der Verhaltensebene formuliert die Person ihr Ziel so spezifisch wie möglich und zerlegt dabei ihr Ziel in viele konkrete Verhaltensweisen. Auf dieser Ebene würde sich Erika ganz genau überlegen, wo sie im Gespräch mit ihrer Chefin sitzen würde, mit welchen Worten sie das Gespräch beginnen und was sie wie sagen würde. Die Formulierung von Zielen auf Verhaltensebene ist auch bekannt unter der SMART-Methode (Spezifisch, Messbar, Attraktiv, Realistisch und Terminiert; Doran 1981; vgl. Locke und Latham 2002). Jahrzehntelange Forschung (z. B. Locke und Latham 2013) empfiehlt, Ziele spezifisch zu formulieren (z. B. ,Ich bitte meine Chefin diesen Mittwoch um $16 \mathrm{Uhr}$ um eine Beförderung in die Abteilung X“), weil damit die Wahrscheinlichkeit steige, das Ziel auch tatsächlich zu erreichen. Bei unspezifisch und vage formulierten Zielen (z. B. „Dieses Jahr spreche ich mit meiner Chefin über eine Beförderung“") sinke die Erfolgswahrscheinlichkeit.

Ziele können jedoch auch auf der Haltungsebene formuliert werden. Diese sind weniger spezifisch, dafür sprechen sie stärker die Person als Ganzes an und steuern damit das Handeln. Um eine Haltung zu entwickeln, formuliert die Person ihr Ziel 
nicht konkret, sondern abstrakt. Das heißt, sie baut damit eine innere Einstellung zum Ziel auf, die sie braucht, um ihr Ziel erreichen zu können (Storch und Krause 2014). Erika würde sich auf dieser Ebene eine neue innere Haltung zum Gespräch mit ihrer Chefin aufbauen, damit sie innerlich in der Lage ist, selbstsicher ihre Argumente für eine Beförderung zu präsentieren. Eine neue innere Haltung aufzubauen, ist deshalb wichtig, weil dadurch unbewusste Bedürfnisse miteinbezogen werden, die oft mit dem bewusst gefassten Ziel auf Verstandesebene in Konflikt stehen. So könnte es beispielsweise sein, dass Erika sich zwar einen beruflichen Aufstieg wünscht (bewusstes Motiv - Verstand), gleichzeitig aber eigentlich mehr Zeit für ihre Familie haben möchte (unbewusstes Bedürfnis).

Unterdrückt der Verstand das Unbewusste, spricht man von Selbstkontrolle (Baumeister 2012). Hier wird eine Absicht gegen unbewusste Impulse, Bedürfnisse und Wünsche durchgesetzt (Baumann und Kuhl 2013). Die Selbstkontrolle kann zwar kurzfristige Erfolge bringen, ist auf Dauer jedoch sehr anstrengend und energieaufwendig und kann dadurch schnell abnehmen. Als Folge werden die bewusst gefassten Absichten nicht umgesetzt. Stellen Sie sich vor, dass Erika bei ihrer Chefin um eine Beförderung anfragt und tatsächlich eine höhere Position antritt. Ihr bewusstes Motiv ist erfüllt, und Erika ist zufrieden. Für die sehr fordernde neue Arbeit muss sie jedoch viele Überstunden machen, wodurch Erika kaum noch Zeit für ihre Familie hat, d.h. ihr unbewusstes Bedürfnis nicht erfüllt ist und sie infolge unglücklich wird. Daher beschließt sie, von der neuen Stelle zurückzutreten und wieder als Kundenberaterin zu arbeiten.

Um solchen negativen Folgen entgegenzuwirken, ist es wichtig, Verstand und Unbewusstes miteinander zu synchronisieren. Dies geschieht durch Selbstregulation. Selbstregulation berücksichtigt und integriert alle für das Selbst relevanten Erfahrungen, wie zum Beispiel Gefühle, Einstellungen und Werte, und bringt diese selbst- und zielrelevanten Informationen mit den eigenen Zielen in Einklang (Baumann und Kuhl 2013; Baumeister und Vohs 2007; Carver und Scheier 1990). Eine solche erfolgreiche Integration erlaubt es einer Person, im Einklang mit dem eigenen Selbst zu handeln, d.h. sich selbstkongruente Ziele zu setzen und umzusetzen (Baumann und Kuhl 2013). Weil die Person in Übereinstimmung mit ihrem Selbst handelt, erlebt sie eine erhöhte Selbstbestimmung, was sie wiederum zu intrinsisch motiviertem Handeln bewegt (Deci und Ryan 2000).

\section{Ziele auf der Haltungsebene: MOTTO-Ziele}

Die Synchronisation von Verstand und Unbewusstem wird durch das Formulieren von Haltungszielen erreicht. Diese Möglichkeit bietet das Zürcher Ressourcen Modell (ZRM; Storch und Krause 2014) mit seiner Vorgehensweise. Hier wird mit zwei Systemen gearbeitet, über die jeder Mensch verfügt und die für die Bildung von Motivation bedeutsam sind - das Intentions- und das Extensionsgedächtnis (Kuhl 2001). Das Intentionsgedächtnis ist das Gedächtnis für bewusste Absichten und Handlungsplanung. Dieses arbeitet genau, aber langsam. Es kann Informationen nur seriell verarbeiten, bedient sich der verbalen Sprache und bewertet Informationen danach, ob sie richtig oder falsch sind. Normen spielen dabei eine große 
Rolle. Beispielsweise sagt Erikas Intentionsgedächtnis ihr, dass es nach ein paar Jahren im Unternehmen wichtig und richtig sei, eine höhere Stelle anzutreten. Das Extensionsgedächtnis enthält autobiographische Erfahrungen, Gefühle, Bedürfnisse, Ziele, Normen und Werte einer Person. Es verarbeitet multikodiert, d.h. es speichert parallel viele bewusste und unbewusste Informationen und Erlebnisse ab und kann die parallel abgespeicherten Informationen auch wieder abrufen. Das Extensionsgedächtnis arbeitet sehr schnell und bewertet mit hoher Geschwindigkeit auf Grundlage von diffusen Körpersignalen (z.B. positives Gefühl), den sogenannten somatischen Markern (Damasio 2003). Diese Bewertung ist entscheidend, ob eine Absicht ausgeführt wird oder nicht. Erika möchte zwar eine Beförderung, hat aber beim Gedanken an den Aufstieg ein mulmiges Gefühl. So würde ihr ihr Körper zeigen, dass ein Konflikt zwischen Bewusstem und Unbewusstem vorhanden ist.

Für das Haltungsziel im ZRM wird mit beiden Systemen gearbeitet. Einerseits reflektieren die Teilnehmenden mithilfe der verbalen Sprache bewusst ihr Ziel, andererseits gleichen sie es mithilfe der nonverbalen Sprache der somatischen Marker laufend mit dem unbewussten System ab. Als Basis für diesen Prozess dient ein Bild, das zu Beginn mit der Frage ausgewählt wird, welches Bild aus einer Auswahl von ressourcenvollen Bildern das stärkste positive Gefühl auslöst. Auf Grundlage dieses Bildes wird dann das Haltungsziel aufgebaut, indem jeder bewusste Schritt mit dem unbewussten System abgeglichen wird. Um das neu gebildete Haltungsziel auch nachhaltig als Teil der eigenen Persönlichkeit zu integrieren, wird es multikodiert abgespeichert, d.h. es wird mithilfe von bestimmten Methoden mit allen Sinnen abgespeichert. Beispielsweise wird das Ziel durch das Embodiment, eine Verkörperung des Ziels, und durch Gegenstände, den sogenannten Erinnerungshilfen, gefestigt. Durch diese Arbeit auf emotionaler, körperlicher, motivationaler und kognitiver Ebene wird der Mensch ganzheitlich angesprochen, und somit wird ein zielrealisierendes Handeln ermöglicht.

Erika wählt das Bild eines hohen Baumes. Ihr Haltungsziel lautet: „Ich bin aufrecht und stehe fest verankert durch die Kraft meiner Wurzeln“. Im Gegensatz zu ihrem ursprünglichen, nie realisierten Silvesterwunsch bekommt sie durch diesen Zielsatz die innere Einstellung und Motivation, ,aufrecht“ ihre Bedürfnisse sehen zu können und „durch die Kraft ihrer Wurzeln“ zu ihnen stehen zu können. Diese Art von Ziel wird im ZRM MOTTO-Ziel genannt. Durch das Bilden des MOTTOZiels wird sich Erika auch ihres unbewussten Bedürfnisses nach Vereinbarkeit von Beruf und Familie bewusst. Dann kann sie dies ihrer Chefin gegenüber kommunizieren und möglicherweise Vorschläge machen, wie sie sich trotz fordernder neuer Arbeit mehr Zeit für ihre Familie nehmen könnte. Beispielsweise könnte sie darum bitten, einen Tag von zuhause aus zu arbeiten, um so auch für die Kinder da sein zu können. Sie würde dann sowohl in der Arbeit als auch im Privaten zufriedener und motivierter sein.

Die Forschung zeigt, dass MOTTO-Ziele im Gegensatz zu spezifischen Zielen die Bindung an ein Ziel stärken (Büche 2017; Weber 2013). Das heißt, dass sich die Person durch ihr MOTTO-Ziel stärker mit dem Ziel identifiziert und sich der Verfolgung dieses Ziels stärker verpflichtet fühlt (Brunstein et al. 2008). Ein MOTTO-Ziel erhöht im Vergleich zu einem spezifischen Ziel auch die erlebte Selbstbestimmung, intrinsische Motivation und optimistisches Denken (Weber 2013) und reduziert das 
subjektive Stresserleben (Storch et al. 2007). Storch und Krause (2014) betonen jedoch, dass MOTTO- und spezifische Ziele nicht als Gegensätze aufgefasst werden sollten, sondern dass sich die beiden Zieltypen ergänzen. Spezifische Ziele eignen sich allerdings eher dann, wenn bereits eine hohe Bindung an das Ziel vorhanden ist, d.h. wenn ein starker Wille bzw. die Entschlossenheit vorhanden ist, das Ziel tatsächlich zu verfolgen, und dieser starke Wille nicht in einem Zielkonflikt mit anderen Zielen in der Außenwelt ist. Ist diese Entschlossenheit noch nicht vorhanden oder herrschen lähmende Zielkonflikte, dann eignen sich MOTTO-Ziele, um diese ins Bewusstsein zu holen und die Zielbindung und damit die Entschlossenheit herzustellen.

\section{Annäherungsmotivation}

Durch die Bildung von MOTTO-Zielen steigt bei Menschen also die Entschlossenheit, ihre Ziele tatsächlich in Angriff zu nehmen. Diese Entschlossenheit wird in der Psychologie auch als Annäherungsmotivation bezeichnet (Harmon-Jones et al. 2011, 2013). Annäherungsmotivation ist eine Motivation, die unser Verhalten auf „Belohnungen“, wie z.B. positive Zielobjekte, Erfahrungen oder Möglichkeiten, richtet (Elliot 2008). Sie entspringt dem Behavioral Approach System (BAS), das menschliches Verhalten steuert und aktiviert, wenn wir die Möglichkeit haben, eine Belohnung zu erhalten (Corr et al. 2013; Gray 1982; Gray und McNaughton 2000). Wenn wir annäherungsmotiviert sind, sind wir voller Energie, fühlen uns stark, fähig und entschlossen und bewegen uns auf ein Ziel zu (Carver und White 1994; Greenaway et al. 2015; Elliot 2008; Harmon-Jones et al. 2011).

Die der Annäherungsmotivation entgegengesetzte Motivation ist die Vermeidungsmotivation. Dies ist eine Motivation, die uns von „Bestrafungen“, wie z.B. negativen Objekten, Erfahrungen oder Möglichkeiten, wegbewegt (Elliot 2008), uns also erst einmal davon abhält, ein Ziel zu verfolgen. Die Vermeidungsmotivation entspringt dem Behavioral Inhibition System (BIS), das durch Zielkonflikte aktiviert wird - Konflikte zwischen etwas, das man erreichen, und etwas, das man vermeiden möchte (Corr et al. 2013; Gray 1982; Gray und McNaughton 2000).

Wenn wir uns Ziele setzen, sind wir erst einmal mit einer Diskrepanz zwischen dem Zustand, den wir anstreben, und dem momentanen Zustand, in dem das Ziel noch nicht erreicht ist, konfrontiert (vgl. Higgins 1989; Jonas et al. 2014). Je nach Zuversicht, diese Diskrepanz überwinden zu können, erleben wir einen Zustand ängstlicher Gehemmtheit (BIS), der sich in ängstlicher Erregung, erhöhter Vigilanz und Hemmung des aktuellen Verhaltens äußert (Corr et al. 2013; Gray und McNaughton 2000; Jonas et al. 2014). 
Stellen Sie sich wieder Erika vor, die sich eine Beförderung wünscht und hinund hergerissen ist, das Gespräch mit ihrer Chefin zu führen (Annäherung) oder es zu vermeiden (aktive Vermeidung). Durch diesen Konflikt wird das BIS ${ }^{1}$ aktiviert, das sich in drei Reaktionen zeigt: (1) Erika wird nervös und ängstlich, wenn sie ihrer Chefin über den Weg läuft (ängstliche Erregung). (2) Erika zeigt besondere Aufmerksamkeit bezüglich riskanten Informationen, die ihre Beförderung gefährden könnten; So hört sie z.B. genau hin, wenn ihre Kollegin und Konkurrentin Karin erzählt, dass sie von ihrer Chefin gelobt wurde (Vigilanz). (3) Erika ist wie gelähmt, wenn es darum geht, einen Termin für das Gespräch mit ihrer Chefin zu vereinbaren, und sie vermeidet jegliches Verhalten, das ihr Ziel gefährden könnte; sie geht also allen Möglichkeiten, ihre Chefin anzutreffen, aus dem Weg, um nicht mit ihr sprechen zu müssen (Verhaltenshemmung). Wenn wir uns länger im BISZustand befinden und es nicht schaffen, aus der Hemmung in die Annäherung zu kommen, steigt die Angst.

Annäherungs- und Vermeidungsmotivation lassen sich nicht nur im Erleben und Verhalten von Menschen nachweisen, sondern auch im Körper messen. Mittels Elektroenzephalografie (EEG) kann die Reaktion des Gehirns auf positive Reize, wie z. B. auf Bilder, in frontozentralen Bereichen gemessen werden (Hajcak et al. 2006). Dies ist ein Indikator dafür, in welchem Ausmaß das Gehirn einen Reiz als Belohnung verarbeitet, und damit ein Maß für Annäherungsmotivation (Angus et al. 2015; Bress und Hajcak 2013; Proudfit 2015). Diese Reaktion wird in den Belohnungszentren des Gehirns generiert (Carlson et al. 2011). Die Forschung zeigt, dass Personen, die sich in einem annäherungsmotivierten Zustand befinden, eine höhere Belohnungsreaktion aufweisen (Mühlberger et al. 2017a). Außerdem zeigen Personen mit annäherungsmotivierten Persönlichkeitseigenschaften (z. B. Extraversion, BASEigenschaften) eine stärkere Belohnungsreaktion (Bress und Hajcak 2013; Cooper et al. 2014; Lange et al. 2012; Smillie et al. 2011) und Personen mit vermeidungsmotivierten Persönlichkeitseigenschaften (z. B. Traurigkeit, depressive Symptome, Ängstlichkeit) eine geringere Belohnungsreaktion (Foti und Hajcak 2009, 2010; Foti et al. 2011; Gu et al. 2010).

\section{Situative und individuelle Unterschiede in der Aktivierung von BIS und BAS}

Bei der Steuerung menschlichen Verhaltens sprechen die beiden Systeme BIS und BAS jeweils bestimmte Bedürfnisse an. Stellen Sie sich z. B. vor, dass Sie bald eine Weltreise antreten. Das BAS wird aktiviert, weil Sie es kaum erwarten können, sich in die vielen neuen Abenteuer zu stürzen, fremde Kulturen kennenzulernen und unbekannte Länder und Orte zu erkunden. Annäherungsmotivation und ein Gefühl

\footnotetext{
1 Das BIS, das einen passiven Vermeidungszustand auslöst, der sich in ängstlicher Erregung, erhöhter Vigilanz und Hemmung des aktuellen Verhaltens äußert, wird durch Zielkonflikte aktiviert. Neben dem BIS nehmen Gray und McNaughton (2000) auch ein aktives Vermeidungssystem - das Fight, Flight, FreezeSystem (FFFS) - an, das durch aversive Reize aktiviert wird. Folglich kommt es zu einer Kampfhandlung, einer Fluchtreaktion oder einem Erstarren, und Panik wird erlebt.
} 
der Vorfreude entsteht. Das BIS wird aktiviert, wenn Belohnungs- und Strafreize gleichzeitig auftauchen. Das heißt, dass Sie sich zwar auf die Reise freuen, aber es könnten Risiken bzw. negative Erlebnisse eintreten. Ihre Vermeidungsmotivation teilt Ihnen daher mit, dass diese möglichst verhindert werden sollten. Das bedeutet, dass uns das BIS davor bewahren kann, Risiken einzugehen und damit Verluste zu machen (Corr et al. 2013; McNaughton und Corr 2004). Die Konsequenz daraus ist, dass Sie z. B. versuchen, die Reise im Vorhinein gut zu planen, eine Karte zur Orientierung und gute Ausrüstung dabeihaben.

Obwohl Menschen beide Motivationen in sich haben, gibt es sowohl individuelle als auch situative Unterschiede in der Ausprägung der beiden Tendenzen (Carver und White 1994; Jonas et al. 2014). Einerseits gibt es Situationen, die stärker das BIS oder das BAS aktivieren. Das BIS wird z.B. dann stärker aktiviert, wenn sich Personen in einer Situation bedroht fühlen und Angst verspüren, etwa dadurch, dass sie an ihre eigene Sterblichkeit erinnert wurden oder die Kontrolle verlieren, d. h. insbesondere dann, wenn sie mit sogenannten existentiellen Bedrohungen konfrontiert werden (vgl. Jonas et al. 2014). So könnte z. B. eine drohende Kündigung einen Kontrollverlust und somit Angst hervorrufen. Sind Personen hingegen in einer Situation auf das Erreichen einer Belohnung hin ausgerichtet und antizipieren sie Freude, sollte bei ihnen das BAS aktiviert sein. Das BAS ist jedoch ebenfalls aktiviert, wenn Personen starken Ärger verspüren (E. Harmon-Jones et al. 2013).

Andererseits gibt es auch dispositionelle Unterschiede zwischen Personen. Annäherungsmotivierte Personen sind stärker handlungsorientiert, entschlossen, reagieren sensibel auf Belohnungen und Gewinne und sehen daher meist die Chancen und Möglichkeiten, die ihnen das Leben bietet. Ihr BAS-System ist reagibler als das von wenig annäherungsmotivierten Persönlichkeiten. Vermeidungsmotivierte Personen reagieren sensibel auf Bestrafungen und Verluste und sehen daher meist die Risiken und Fehler, die sie begehen könnten. Ihr BIS-System ist reagibler als das von wenig vermeidungsmotivierten Personen. Sie sind eher ängstlich und nervös und kommen nur schwer aus diesem negativen Zustand heraus (Carver und White 1994; Stemmler et al. 2016). Diese dispositionellen Unterschiede beeinflussen, wie sensibel Menschen auf Belohnungen und Bestrafungen reagieren und wie schnell sie daher vom BIS-Zustand in den BAS-Zustand wechseln können.

Fortführung des Fallbeispiels Sehen wir uns die Persönlichkeit von Erika, die seit mehr als 5 Jahren als Kundenberaterin tätig ist, nun genauer an. Erika beschreibt sich selbst als eine generell eher unsichere, besorgte Person, die Angst davor hat, Fehler zu machen. Wenn ihr etwas Unangenehmes, wie z. B. das Gespräch mit ihrer Chefin, bevorsteht, ist sie ziemlich unruhig. Sie reagiert sehr sensibel auf potentielle „Gefahren“ und hat viele Ängste. Kritik verletzt sie sehr stark, und sie schafft es dann nur schwer, aus diesem negativen Erleben herauszukommen. Sie ist oft nervös, eher passiv als handlungsorientiert und zeigt geringes Vertrauen in ihre eigenen Fähigkeiten, ihre Ziele zu erreichen. Erikas Kollegin Karin, die ebenfalls seit mehr als 5 Jahren in demselben Versicherungsunternehmen als Kundenberaterin tätig ist, beschreibt sich selbst als eine sehr handlungsorientierte Person, die ihre Ziele hartnäckig verfolgt. Sie reagiert sensibel auf potentielle Belohnungen und strengt sich dann besonders an, diese zu erhalten. Sie ist voller Energie, wenn es darum geht, ihre Ziele 
$z u$ verfolgen, und zeigt hohes Vertrauen in ihre eigenen Fähigkeiten, diese auch tatsächlich zu erreichen. Während Erika dem Prototypen der vermeidungsmotivierten Persönlichkeit zuzuordnen ist, ist Karin dem Prototypen der annäherungsmotivierten Persönlichkeit zuzuordnen.

Auch Erikas Kollegin Karin hat das Ziel, bei ihrer Chefin um eine Beförderung anzufragen. Da Erika und Karin das Gespräch schon länger vor sich herschieben, beschließen die beiden, an einem Training zu Gesprächsführung teilzunehmen. Nach dem Training ist die vermeidungsmotivierte Erika sehr zufrieden, hat das Gefühl, ihrem Ziel näher gekommen zu sein, und ist entschlossen, das Gespräch mit ihrer Chefin zu führen. Die annäherungsmotivierte Karin hingegen ist nach dem Training stark verunsichert und ahnt bereits jetzt, dass sie das Gespräch nicht führen wird.

Woran kann es nun liegen, dass das Training bei Erika erfolgreich, bei Karin weniger erfolgreich war?

\section{Ziele passend zur Persönlichkeit formulieren - empirische Überprüfung}

Die psychologische Forschung zeigt, dass eine Passung zwischen der Persönlichkeit und der Strategie zur Erreichung der eigenen Ziele zentral ist, da sich eine Passung für Menschen stimmig anfühlt und sie dann motiviert sind, ihr Ziel tatsächlich in Angriff zu nehmen (vgl. Böhm et al. 2017; Cesario und Higgins 2008). So wurden in einer Studienreihe (Böhm et al., 2017) z. B. Handlungsstrategien entweder mithilfe einer Annäherungsstrategie (,Welche Handlungen sind notwendig, um deine Ziele zu erreichen?") oder mithilfe einer Vermeidungsstrategie formuliert (,Welche Handlungen solltest du vermeiden, um deine Ziele zu erreichen?“). Die zur jeweiligen Persönlichkeit passende Zielformulierung führte zu einer erhöhten intrinsischen Zielmotivation und zu mehr Selbstwirksamkeit. Um diese positiven Effekte zu erreichen, ist die Passung v. a. für Beratungsformate wie Coaching oder Training zentral, und diese beginnt bereits bei der Formulierung des Ziels.

Dass nicht jede Art von Zielformulierung für jeden Persönlichkeitstyp passend ist, zeigt z. B. eine Studie, in der Studierende an einem Coaching zur Reduktion von Prokrastination, d. h. Lernaufschiebeverhalten, teilnahmen (Mühlberger et al. 2017c). Dabei formulierten die Studierenden ein lernbezogenes Ziel zuerst spezifisch, d.h. sie zerlegten ihr Ziel in viele konkrete Verhaltensweisen (Formulierung des Ziels auf Verhaltensebene). Danach besprachen sie ihr Ziel mit einem Coach. Obwohl alle Studierenden davon überzeugt waren, ihrem Ziel durch das Coaching näher gekommen zu sein, berichteten nur annäherungsmotivierte Personen nach dem Coaching weniger Prokrastination. Vermeidungsmotivierte Personen berichteten hingegen mehr Prokrastination. Dies war darauf zurückzuführen, dass die vermeidungsmotivierten Studierenden ihre definierten Ziele nicht als ihre eigenen Ziele betrachteten, sondern diese mehr fremd- als selbstbestimmt erlebten. Das bedeutet, dass sich die Ziele für sie nicht stimmig anfühlten und es daher zu keiner Passung zwischen ihrer Persönlichkeit und der Strategie zur Zielerreichung kam. Um also Ziele so zu formulieren, dass sie auch handlungswirksam werden, sollte die Zielformulierung zur Persönlichkeit passen. Die spezifische Zielformulierung auf Verhaltensebene scheint bei stark 
vermeidungsmotivierten Personen nicht effektiv zu sein. Welche Zielformulierung braucht es bei diesen Personen, damit auch sie ihre Ziele verfolgen?

Wir untersuchten diese Fragestellung in einer Studie (Büche 2017; Mühlberger et al. 2017b), in der wir die Zielformulierung auf Verhaltensebene (SMART) mit der Zielformulierung auf Haltungsebene (MOTTO) miteinander verglichen. Dazu nahmen Personen an einem SMART- oder MOTTO-Training zur effektiven Gesprächsführung teil. Die Teilnehmenden formulierten ihre Ziele entweder nach den SMART-Kriterien oder nach den Kriterien des ZRM. Beide Trainingsgruppen wählten sich passend zu ihrem Ziel ein Bild. Im MOTTO-Training wählten sich die Teilnehmenden zu Beginn ein Bild, um sich damit ihr Haltungsziel zu erarbeiten. Im SMART-Training wählten sie ein Bild, das ihr Ziel auf Verhaltensebene repräsentierte. Wir untersuchten, wie die selbstgewählten Bilder auf die Zielmotivation der Trainingsteilnehmenden wirkten, wenn diese das Ziel hatten, ein schwieriges Gespräch zu führen. Dafür erfassten wir mittels Fragebögen, inwiefern die Teilnehmenden sich mit ihrem Ziel identifizierten (Zielbindung), inwiefern sie sich erwarteten, aufgrund eigener Fähigkeiten gewünschte Handlungen erfolgreich ausführen zu können (Selbstwirksamkeitserwartung), und inwiefern sie das Gespräch als Herausforderung anstatt als Bedrohung wahrnahmen (Herausforderungserleben). Im Vergleich zu Personen des SMART-Trainings zeigten Personen des MOTTO-Trainings einen Monat nach dem Training mehr Zielbindung (vgl. Weber 2013), eine höhere Selbstwirksamkeitserwartung und nahmen das schwierige Gespräch stärker als positive Herausforderung wahr.

Mittels Elektroenzephalografie (EEG) erfassten wir außerdem die Hirnaktivität in frontozentralen Hirnbereichen, wenn die Teilnehmenden ihre selbstgewählten Bilder betrachteten. Die Messung in diesen Bereichen gibt Aufschluss über die erlebte Annäherungsmotivation (z. B. Becker et al. 2014; Carlson et al. 2015; Proudfit 2015), d.h. die Entschlossenheit, ihr Ziel in Angriff zu nehmen. Dafür kamen die Teilnehmenden ungefähr eine Woche nach den Trainings ins Labor und sahen auf dem Computerbildschirm ihr selbstgewähltes Bild, das sie entweder an ihr MOTTO- oder ihr SMART-Ziel erinnern sollte. Hier zeigte sich ein Unterschied zwischen Personen, die sich vor den Trainings im BIS befanden, d.h. stark verunsichert, gehemmt, nervös, besorgt, unruhig und ängstlich waren, und Personen, die sich vor den Trainings nicht im BIS befanden. Während die stärker besorgten Teilnehmenden (hohes BIS) zu mehr Annäherungsmotivation tendierten, wenn sie am MOTTO-Training als wenn sie am SMART-Training teilgenommen hatten, tendierten die wenig Besorgten (kein BIS) zum Erleben höherer Annäherungsmotivation, wenn sie am SMARTTraining als wenn sie am MOTTO-Training teilgenommen hatten. Besonders interessant war, dass Personen, die sich im BIS befanden und dann am MOTTO-Training teilnahmen, nach dem Training mehr Annäherungsmotivation erlebten als Personen, die sich nicht im BIS befanden und dann am MOTTO-Training teilnahmen. Das heißt, dass Besorgte vom MOTTO-Training stärker profitierten als wenig Besorgte. Die Ergebnisse deuten darauf hin, dass Haltungsziele bei besorgten Personen stärker wirken als bei wenig besorgten Personen. Bei wenig besorgten Personen scheint SMART im Gegensatz zu MOTTO besser zu wirken. 
Zusammengefasst bedeutet dies, dass die Zielformulierung alleine nicht unbedingt ausschlaggebend für Erfolg ist, sondern zusätzlich die Passung zur Persönlichkeit eine zentrale Rolle spielt. Ist eine Person grundsätzlich wenig besorgt und bereits mehr im annäherungsmotivierten Zustand, ist sie bereits fest entschlossen und davon überzeugt, ihr Ziel in Angriff nehmen zu können. Sie identifiziert sich mit ihrem Ziel, d.h. die Zielbindung ist bereits vorhanden. Eine SMARTe Zielformulierung mit konkreter Planung des „Wann, Wo, Wie?“ scheint bei dieser Person zielführend zu sein. Ist eine Person jedoch grundsätzlich eher vermeidungsmotiviert, d.h. steckt sie noch in der Unsicherheit fest, ist sie wenig entschlossen und kommt nur schwer ins Handeln. Die Zielbindung und damit die Entschlossenheit, das Ziel in Angriff zu nehmen, muss erst hergestellt werden. Eine Zielformulierung mithilfe von MOTTO-Zielen scheint bei dieser Person erfolgversprechend zu sein. Erst dann ist eine konkrete Planung des zu erreichenden Ziels, z.B. anhand der SMART-Kriterien, sinnvoll.

\section{Bedeutung für die Praxis und Fazit}

Bleibt nun die Frage, was dies für die Coachingpraxis im Einzelsetting bedeutet, wenn es so wichtig für die erfolgreiche Zielerreichung ist, dass die Zielformulierung passend zur Persönlichkeit ausgewählt wird. Stellen Sie sich vor, ein Coach hätte mit Erika sofort für ihren ersten Wunsch SMART formulieren lassen und sie damit zurück ,,ins Leben geschickt““. Im besten Fall hätte sie das Gespräch mit ihrer Chefin wieder nicht geführt. Hätte sie es geführt, wäre sie sogar erfolgreich gewesen, könnte den Erfolg aber nicht wirklich genießen. Das Thema der Beförderung wäre zwar gelöst, ihr inneres „Mutterherz“ jedoch im Dauerkonflikt. Somit wäre das eigentliche unbewusste Thema, wie sie in der derzeitigen Lebenssituation die Balance zwischen Arbeit und Familie schaffen kann, unbearbeitet geblieben.

Da die Formulierung des Ziels meist bereits zu Beginn eines Beratungsprozesses stattfindet und Beratende ihre Klient/innen erst im Laufe des Beratungsprozesses genauer kennenlernen, stellt sich die Frage, wie Beratende die motivationale Orientierung am besten herausfinden. Unseres Erachtens ist gerade in der Phase der Auftragsklärung eine hohe Sensibilität für die Persönlichkeit des Klienten bzw. dessen besondere Situation und die damit verbundene motivationale Orientierung im Gespräch notwendig. Zusätzlich könnten Beratende neben dem Einsatz diagnostischer Instrumente (z. B. die BIS/BAS Skalen von Carver und White 1994), die im Beratungssetting aber wohl eher selten angewendet werden, auch mit Ankertechniken den Persönlichkeitsstil und/oder die Zielbindung und die Annäherungsmotivation der Person herausarbeiten. Dazu legt man mit Kärtchen das Jetzt, das Ziel und die Zeit unmittelbar nach dem erwünschten Ziel auf. Der/die Klient/in fühlt sich in die entsprechende Situation ein. Wenn im Beispiel von Erika nach dem erreichten Ziel ihre Mimik, Gestik und ihre Körperhaltung ressourcenvoll sind und ihre Beschreibung vom erlebten Zustand positiv, kann man daraus schließen, dass ihr erwünschtes Ziel kongruent zu ihrer Persönlichkeit und ihrer derzeitigen Lebenssituation ist. In diesem Fall könnte man sofort mit der spezifischen Handlungsplanung beginnen und das Ziel SMART beschreiben und planen lassen. Ist Erika hingegen ängstlich 
und sorgenvoll zweifelnd auf dem Anker nach dem Ziel und scheint es noch keine oder nur eine ambivalente Zielbindung zu geben, was sich wiederum an der Mimik, an der Körperhaltung und ihren Aussagen ablesen lässt, dann kann für Erika eine Zielformulierung auf der Haltungsebene entscheidend sein, um in die kongruente Zielbindung und in die Annäherungsmotivation zu kommen. Hat Erika dann eine neue Haltung dem Ziel gegenüber aufgebaut und eventuelle Zielanpassungen vorgenommen, kann das Ziel schließlich SMART vorbereitet werden.

So gesehen ist es für Coaches sehr wichtig, zuerst zu klären, welchen Persönlichkeitsstil die Klient/innen haben und ob eine Zielbindung und Annäherungsmotivation schon gegeben ist oder ob die Klient/innen noch in einer Vermeidungsmotivation sind und/oder das Ziel noch nicht kongruent zur Persönlichkeit ist. Wenn dies geklärt ist, hat man zwei Möglichkeiten: (1) Der/Die Klient/in klärt mit Hilfe eines MOTTOZiels sein erwünschtes Ziel und baut durch die Erarbeitung einer neuen Haltung eine hohe Zielbindung und Annäherungsmotivation für dieses auf. (2) Wenn offensichtlich die Annäherungsmotivation schon gegeben ist, kann man sofort SMART mit der Vorbereitung für das Ziel beginnen. Die Zielformulierung passend zur Persönlichkeit auszuwählen, spielt also eine entscheidende Rolle dabei, ob wir frühzeitig aufgeben oder unsere Ziele auch tatsächlich weiterverfolgen.

Funding Open access funding provided by Paris Lodron University of Salzburg.

Open Access Dieser Artikel wird unter der Creative Commons Namensnennung 4.0 International Lizenz (http://creativecommons.org/licenses/by/4.0/deed.de) veröffentlicht, welche die Nutzung, Vervielfältigung, Bearbeitung, Verbreitung und Wiedergabe in jeglichem Medium und Format erlaubt, sofern Sie den/die ursprünglichen Autor(en) und die Quelle ordnungsgemäß nennen, einen Link zur Creative Commons Lizenz beifügen und angeben, ob Änderungen vorgenommen wurden.

\section{Literatur}

Angus, D.J., Kemkes, K., Schutter, D.J.L.G., \& Harmon-Jones, E. (2015). Anger is associated with reward-related electrocortical activity: evidence from the reward positivity. Psychophysiology, 52, 1271-1280. https://doi.org/10.1111/psyp.12460.

Baumann, N., \& Kuhl, J. (2013). Selbstregulation und Selbstkontrolle. In W. Sarges (Hrsg.), ManagementDiagnostik (Bd. 4, S. 263-271). Göttingen: Hogrefe.

Baumeister, R. (2012). Die Macht der Disziplin. Frankfurt a.M.: Campus.

Baumeister, R. F., \& Vohs, K. D. (2007). Self-regulation, ego depletion, and motivation. Social and Personality Psychology Compass, 1(1), 115-128. https://doi.org/10.1111/j.1751-9004.2007.00001.x.

Becker, M.P.I., Nitsch, A.M., Miltner, W.H.R., \& Straube, T. (2014). A single-trial estimation of the feedback-related negativity and its relation to BOLD responses in a time-estimation task. The Journal of Neuroscience, 34, 3005-3012. https://doi.org/10.1523/JNEUROSCI.3684-13.2014.

Böhm, A., Mühlberger, C., \& Jonas, E. (2017). Wachstums- und Sicherheitsorientierung im Coaching Erfolg durch motivationale Passung. In S. Greif, H. Möller \& W. Scholl (Hrsg.), Handbuch Schlüsselkonzepte im Coaching. Heidelberg: Springer.

Bress, J.N., \& Hajcak, G. (2013). Self-report and behavioral measures of reward sensitivity predict the feedback negativity. Psychophysiology, 50(7), 610-616. https://doi.org/10.1111/psyp.12053.

Brunstein, J.C., Dargel, A., Glaser, C., Schmitt, C.H., \& Spörer, N. (2008). Persönliche Ziele im Studium. Erprobung einer Intervention zur Steigerung der Zieleffektivität und Zufriedenheit im Studium. Zeitschrift für Pädagogische Psychologie, 22, 177-191.

Büche, A. (2017). Ressourcenvoll riskante Gespräche führen. Masterarbeit, Universität Salzburg.

Carlson, J.M., Foti, D., Mujica-Parodi, L.R., Harmon-Jones, E., \& Hajcak, G. (2011). Ventral striatal and medial prefrontal BOLD activation is correlated with reward-related electrocortical activity: a 
combined ERP and fMRI study. NeuroImage, 57, 1608-1616. https://doi.org/10.1016/j.neuroimage. 2011.05.037.

Carlson, J. M., Foti, D., Harmon-Jones, E., \& Proudfit, G. H. (2015). Midbrain volume predicts fMRI and ERP measures of reward reactivity. Brain Structure and Function, 220, 1861-1866. https://doi.org/ 10.1007/s00429-014-0725-9.

Carver, C.S., \& Scheier, M.F. (1990). Origins and functions of positive and negative affect: a controlprocess view. Psychological Review, 97(1), 19-35.

Carver, C.S., \& White, T.L. (1994). Behavioral inhibition, behavioral activation, and affective responses to impending reward and punishment: the BIS/BAS scales. Journal of Personality and Social Psychology, 67(2), 319-333. https://doi.org/10.1037//0022-3514.67.2.319.

Cesario, J., \& Higgins, E.T. (2008). Making message recipients „feel right“: how nonverbal cues can increase persuasion. Psychological Science, 19(5), 415-420.

Cooper, A. J., Duke, E., Pickering, A.D., \& Smillie, L.D. (2014). Individual differences in reward prediction error: contrasting relations between feedback-related negativity and trait measures of reward sensitivity, impulsivity and extraversion. Frontiers in Human Neuroscience. https://doi.org/10.3389/ fnhum.2014.00248.

Corr, P. J., DeYoung, C. G., \& McNaughton, N. (2013). Motivation and personality: a neuropsychological perspective. Social and Personality Psychology Compass, 7(3), 158-175. https://doi.org/10.1111/ spc3.12016.

Damasio, A. R. (2003). Ich fühle also bin ich. Die Entschlüsselung des Bewusstsein. München: List.

Deci, E.L., \& Ryan, R.M. (2000). The „what“ and „why“ of goal pursuits: human needs and the selfdetermination of behavior. Psychological Inquiry, 11(4), 227-268.

Doran, G. T. (1981). There's a S.M.A.R.T. way to write management's goals and objectives. Management Review, 70(11), 35-36.

Elliot, A. J. (Hrsg.). (2008). Handbook of approach and avoidance motivation. New York: Taylor \& Francis.

Foti, D., \& Hajcak, G. (2009). Depression and reduced sensitivity to non-rewards versus rewards: evidence from event-related potentials. Biological Psychology, 81(1), 1-8. https://doi.org/10.1016/j.biopsycho. 2008.12.004.

Foti, D., \& Hajcak, G. (2010). State sadness reduces neural sensitivity to nonrewards versus rewards. Neuroreport, 21(2), 143-147. https://doi.org/10.1097/WNR.0b013e3283356448.

Foti, D., Kotov, R., Klein, D. N., \& Hajcak, G. (2011). Abnormal neural sensitivity to monetary gains versus losses among adolescents at risk for depression. Journal of Abnormal Child Psychology, 39(7), 913-924. https://doi.org/10.1007/s10802-011-9503-9.

Gray, J. A. (1982). The neuropsychology of anxiety. New York: Oxford University Press.

Gray, J.A., \& McNaughton, N. (2000). The neuropsychology of anxiety: an enquiry in to the functions of the septo-hippocampal system (2. Aufl.). Oxford: Oxford University Press.

Greenaway, K.H., Storrs, K. R., Philipp, M.C., Louis, W. R., Hornsey, M. J., \& Vohs, K. D. (2015). Loss of control stimulates approach motivation. Journal of Experimental Social Psychology, 56, 235-241. https://doi.org/10.1016/j.jesp.2014.10.009.

Gu, R., Ge, Y., Jiang, Y., \& Luo, Y.J. (2010). Anxiety and outcome evaluation: the good, the bad and the ambiguous. Biological Psychology, 85(2), 200-206. https://doi.org/10.1016/j.biopsycho.2010.07. 001.

Hajcak, G., Moser, J.S., Holroyd, C. B., \& Simons, R. F. (2006). The feedback-related negativity reflects the binary evaluation of good versus bad outcomes. Biological Psychology, 71(2), 148-154. https:// doi.org/10.1016/j.biopsycho.2005.04.001.

Harmon-Jones, C., Schmeichel, B.J., Mennitt, E., \& Harmon-Jones, E. (2011). The expression of determination: similarities between anger and approach-related positive affect. Journal of Personality and Social Psychology, 100, 172-181.

Harmon-Jones, E., Harmon-Jones, C., \& Price, T. F. (2013). What is approach motivation? Emotion Review, 5, 291-295.

Higgins, E. T. (1989). Self-discrepancy theory: What patterns of self-beliefs cause people to suffer? Advances in Experimental Social Psychology, 22, 93-136.

Jonas, E., McGregor, I., Klackl, J., Agroskin, D., Fritsche, I., Holbrook, C., \& Quirin, M. (2014). Threat and defense: from anxiety to approach. In J.M. Olson \& M.P. Zanna (Hrsg.), Advances in experimental social psychology (S. 219-286). San Diego: Academic Press.

Kuhl, J. (2001). Motivation und Persönlichkeit. Interaktion psychischer Systeme. Göttingen: Hogrefe. 
Lange, S., Leue, A., \& Beauducel, A. (2012). Behavioral approach and reward processing: results on feedback-related negativity and P3 component. Biological Psychology, 89(2), 416-425. https://doi. org/10.1016/j.biopsycho.2011.12.004.

Locke, E., \& Latham, G. (2013). New developments in goal setting and task performance. New York: Routledge.

Locke, E. A., \& Latham, G.P. (2002). Building a practically useful theory of goal setting and task motivation. American Psychologist, 57(9), 705-717.

McNaughton, N., \& Corr, P. J. (2004). A two-dimensional neuropsychology of defense: fear/anxiety and defensive distance. Neuroscience and Biobehavioral Reviews, 28(3), 285-305. https://doi.org/10. 1016/j.neubiorev.2004.03.005.

Mühlberger, C., Angus, D. J., Jonas, E., Harmon-Jones, C., \& Harmon-Jones, E. (2017a). Perceived control increases the reward positivity and stimulus preceding negativity. Psychophysiology, 54(2), 310-322. https://doi.org/10.1111/psyp.12786.

Mühlberger, C., Büche, A., Weber, J., \& Jonas, E. (2017b). MOTTO vs. SMART. Unveröffentlichte Daten. Universität Salzburg.

Mühlberger, C., Mühlberger, M., Traut-Mattausch, \& Jonas, E. (2017c). Stop or Go? Autonomieerleben im Coaching und der Einfluss der BIS/BAS-Persönlichkeit. Unveröffentlichte Daten. Universität Salzburg.

Proudfit, G. H. (2015). The reward positivity: from basic research on reward to a biomarker for depression. Psychophysiology, 52(4), 449-459. https://doi.org/10.1111/psyp.12370.

Smillie, L.D., Cooper, A.J., \& Pickering, A.D. (2011). Individual differences in reward-prediction-error: extraversion and feedback-related negativity. Social Cognitive and Affective Neuroscience, 6(5), 646-652. https://doi.org/10.1093/scan/nsq078.

Stemmler, G., Hagemann, D., Amelang, M., \& Spinath, F. M. (2016). Differentielle Psychologie und Persönlichkeitsforschung (8. Aufl.). Stuttgart: Kohlhammer.

Storch, M. (2008). Rauchpause. Wie das Unbewusste dabei hilft, das Rauchen zu vergessen. Bern: Huber.

Storch, M., \& Krause, F. (2014). Selbstmanagement - ressourcenorientiert. Grundlagen und Trainingsmanual für die Arbeit mit dem Zürcher Ressourcen Modell (ZRM) (5. Aufl.). Bern: Huber.

Storch, M., Gaab, J., Küttel, Y., Stüssi, A., \& Fend, H. (2007). Psychoneuroendocrine effects of resourceactivating stress management training. Health Psychology, 26, 456-463.

Weber, J. (2013). Turning Duty into Joy! Optimierung der Selbstregulation durch Motto-Ziele. Dissertation, Universität Osnabrïck.

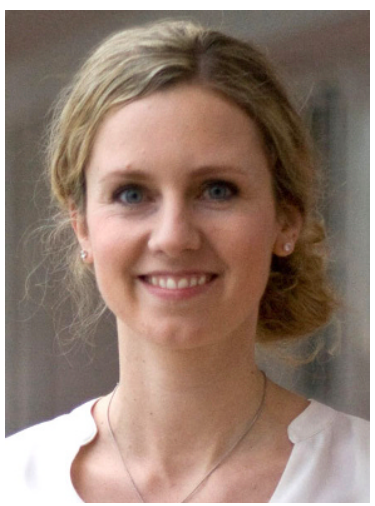

Mag. Dr. Christina Mühlberger Postdoc der Abteilung Sozialpsychologie, Universität Salzburg, Zertifizierte Coach, Zertifizierte ZRMTrainerin, Schwerpunkte: Soziale Interaktionen, Beratungsformate (v. a. Coaching und Training), Erfüllung und Verletzung psychologischer Bedürfnisse, Motivationsprozesse (Annäherungsmotivation, Regulatorischer Fokus, Selbstregulationsprozesse), Perspektivenübernahme und Empathie. Internet: https://www.uni-salzburg/sozialpsychologie. 

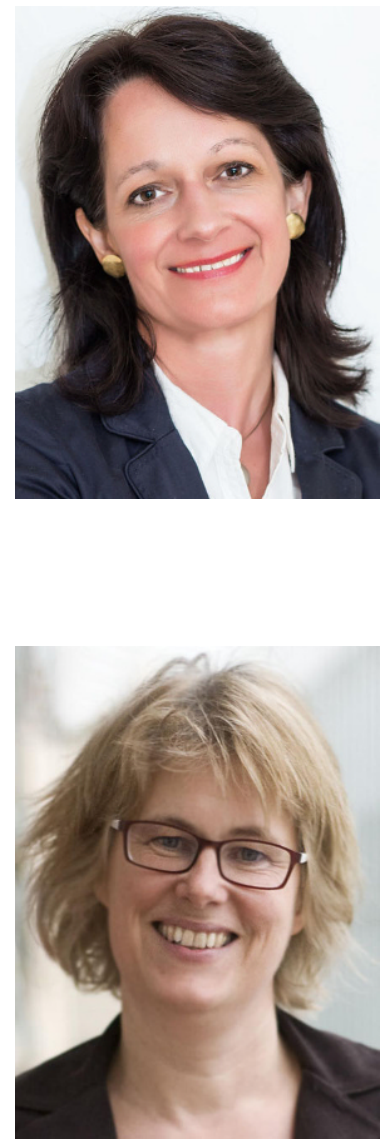

Angela Büche MSc. Supervision, Mediation und Coaching, ZRMTrainerin, Lehrbeauftragte an Universitäten, Musikschulen, Ministerien, Cellistin. Eigene Beratungspraxis in Salzburg und Wien, tätig im Team der: ÖVS Sbg (Österreichische Vereinigung der SupervisorInnen); ÖGFMM (Österreichische Gesellschaft für Musik und Medizin); AFNB (Akademie für Neurowissenschaftliches Bildungsmanagement). Schwerpunkte: Motivations- und Selbstmanagementtraining, Lernpsychologie, Embodied Communication, Coaching für Projektleiter/innen, Personalentwicklung 4.0. Internet: https://www.stimmig-leben.com.

Prof. Dr. Eva Jonas Professorin und Leiterin der Abteilung Sozialpsychologie, Universität Salzburg, Leiterin des Universitätslehrgangs „Master in Training and Development" der Salzburg Management Business School (SMBS) an der Universität Salzburg. Schwerpunkte: Motivierte soziale Kognition, Umgang mit Bedrohungen, Gestaltung funktionaler und dysfunktionaler Interaktionen, Analyse verschiedener Beratungsformate (Coaching, Mentoring, Supervision, Mediation), Perspektivenübernahme und Empathie. Internet: https://www.uni-salzburg/ sozialpsychologie. 